\title{
Effects of Anwei decoction on the protein expression of TFF in rats with chronic atrophic gastritis
}

\author{
Wei Wei ${ }^{1}$, Shouning Lin $^{2 *}$, Yongping $\mathrm{Zhu}^{2}$ \\ ${ }^{1}$ Guangxi University of Chinese Medicine, Nanning, China \\ ${ }^{2}$ Digestive System Department, Ruikang Hospital Affiliated to Guangxi University of Chinese Medicine, Nanning, China; \\ *Corresponding Author: LSN77766@sina.com
}

Received 15 October 2013; revised 10 November 2013; accepted 18 November 2013

Copyright (C) 2014 Wei Wei et al. This is an open access article distributed under the Creative Commons Attribution License, which permits unrestricted use, distribution, and reproduction in any medium, provided the original work is properly cited. In accordance of the Creative Commons Attribution License all Copyrights (C) 2014 are reserved for SCIRP and the owner of the intellectual property Wei Wei et al. All Copyright (C) 2014 are guarded by law and by SCIRP as a guardian.

\section{ABSTRACT}

Objective: To explore the effects of Anwei decoction (AD) on the protein expression of TFF in rats with chronic atrophic gastritis(CAG). Methods: Forty-eight healthy rats were randomly divided into 4 groups: normal control group, pathologic model group, Anwei Decoction group, and Weifuchun group. CAG was induced in rats with $\mathrm{N}$-methy-N-nitro-N-nitrogua-nidine (MNNG). The protein expression of TFF in rats' gastric mucosa was determined by immunohistochemistry. Results: Compared with that in the normal control group, the protein expression of TFF1 was significantly enhanced in the pathologic model, Anwei Decoction and Weifuchun groups (both $P<0.01$ ). The protein expression level of TFF1 was significantly higher in the Anwei Decoction group than in the Weifuchun group $(P<$ 0.01). Compared with the normal control group, the protein expression of TFF2 was significantly enhanced in the pathologic model, Anwei Decoction and Weifuchun groups (both $P<0.01$ ). The protein expression level of TFF2 was significantly higher in the Anwei Decoction group than in the Weifuchun group $(P<0.01)$. In comparison with the pathologic model group, the protein expression of TFF3 was remarkably reduced in Anwei Decoction and Weifuchun groups (both $P<0.01$ ). but there was no difference between the group of Anwei decoction and the group of Weifuchun $(P>0.05)$. Conclusion: Anwei decoction may be effective in the treatment of CAG by enhancing the protein expression of TFF1, TFF2 while reducing that of TFF3 in gastric mucosas.

\section{KEYWORDS}

Anwei Decoction; Atrophic Gastritis; TFF1; TFF2; TFF3; Experimental Research

\section{INTRODUCTION}

Chronic atrophic gastritis (CAG), a type of common digestive diseases, is closely related to gastric cancers. The relationship between antral atrophic gastritis and intestinal-typed gastric cancer has been confirmed. The World Health Organization has defined CAG as precancerous diseases, so the control of CAG is of great significance. Chinese medicine treatment can broaden the therapeutic approach of chronic gastritis [1]. Trefoil peptide family (trefoil factor family, TFF) is a group of small molecule polypeptides with a specific trefoil domain synthesized and secreted by the gastrointestinal mucus-secretion epithelia. In mammals there are three factors: breast cancer-related peptide (TFF1/PS2), spasmolytic polypeptide (TFF2/SP) and intestinal trefoil factor (TFF3/ITF). TFF has a strong ability to promote proliferation and migration of epithelial cells. It can promote the reconstruction of the damaged area and accelerate epithelial cell migration speed [2]. The compound Chinese medicine Anwei decoction is a proved recipe for CAG treatment formulated by Professor Lin Peixiang, a famous Chinese doctor of traditional Chinese medicine. Despite its significant clinical effects [3], the mechanism concerning CAG treatment of Anwei decoction is not fully understood. In this paper, we conducted animal experiments to observe the effect of Anwei decoction on gastric TFF expression and explore its mechanism in the prevention and treatment of CAG. 


\section{MATERIALS AND METHODS}

\subsection{Materials}

Healthy Wistar rats of clean grade, $\hat{\partial}$, body weight 140 - 170 g, were offered by the Experimental Animal Center of Guangxi Institute for Food and Drug Control (animal Certificate No.: SCXX Gui 2003-0001). Anwei decoction was made from Chinese medicine granules, whose main components included Pinellia, berberine, ginger, aggregata, salvia, lily, peony root, Coix seed, roasted liquorice (13:5:5:7:15: 20:20:10:5). These granules were produced by Tianjiang Pharmaceutical Co., Ltd., Jiangyin, Jiangsu, and bought from Ruikang Affiliated Hospital of Guangxi Traditional Chinese Medical University. Weifuchun tablets were produced by $\mathrm{Hu}$ Yuqing Tang Pharmaceutical Co. Ltd., Hangzhou, Zhejiang (Lot No.: Guoyao Zhunzi Z20040003; product specification: $0.359 \mathrm{~g}$ /tablet), and also purchased from Ruikang Affiliated Hospital of Guangxi Traditional Chinese Medical University. Immunohistochemical reagents TFF1, TFF2, TFF3 polyclonal antibodies were purchased from Orson Biotechnology Co., Ltd.; SP immunohistochemistry kit Beijing Zhongshan Golden Bridge Biotechnology Co., Ltd.. Frozen section machine: British Thermo Elecron Corporation production; TP1020 automatic biological tissue dewatering machines, EGll40H paraffin embedding machine, HM 355 S-type paraffin slicing machine: Germany Diamicron (MICROM) company; YT-6 biological tissue spread baking sheet machine: Xiaogan matt medical electronic technology; electric heated blast drying oven: Shanghai Yuejin Medical Instrument Factory production; BX50F-3 binocular microscope (with automatic camera device NikonD700): Japan Olypus company.

\subsection{Methods}

\subsubsection{Grouping and Administration}

Forty-eight rats were randomly divided into 4 groups $(n=12)$ : non control group (without any treatment, normal feeding), pathological model group, Anwei decoction group, Weifuchun control group; the modeling was done by following the literature [4]. Three groups (pathological model group, Anwei decoction group, Weifuchun control group ) were free to drink $170 \mathrm{mg} / \mathrm{L}$ of MNNG solution, not accessible to other drinking water during the modeling period and supplied with common fodder for a total of 8 weeks. Eight weeks later, 2 rats were randomly selected and killed in each group, their stomachs were taken out and pathological examination was conducted to determine whether the models were successful. Once the success of modeling was confirmed, gastric perfusion started: Pathological model group were fed with $4 \mathrm{~mL} / \mathrm{d}$ of pure water; Anwei decoction group were fed with Anwei decoction suspension $(2.5 \mathrm{~kg} / \mathrm{L})$ at a do- sage of $1 \mathrm{~mL}$ per $100 \mathrm{~g}$ of body weight; Weifuchun group were fed with Weifuchun suspension $(0.29 \mathrm{~g} / 4 \mathrm{~mL})$; all feeding was done once a day and lasted for 4 weeks.

\subsubsection{Specimen Preparation}

After being administrated for 4 weeks, rats in each group entered a $24 \mathrm{~h}$ fast. They were anaesthetized by administering $1 \%$ urethane solution $(1 \mathrm{~mL} / 100 \mathrm{~g}$ of body weight) through subcutaneous injection, their gastric tissues were taken out and subjected to further studies. The external form and structure of the gastric tissue were observed. The stomach was dissected along its lesser curvature, the gastric mucosa was washed with normal saline and flattened so that its form, structure and color could be observed. A piece of gastric mucosa about 0.5 $\mathrm{cm} \times 0.5 \mathrm{~cm}$ in size and a piece of gastric antrum were cut along the direction from the gastric antrum (near the pyloric ostium) to the greater gastric curvature and fixed in $10 \%$ formaldehyde solution to make paraffin sections. The paraffin sections were subjected to HE staining and immunohistochemical tests. Another small piece of gastric tissue was stored at $-80^{\circ} \mathrm{C}$ in the refrigerator for future use.

Paraffin sections $10 \%$ formaldehyde 120 min- gradient of alcohol $(80 \% \rightarrow 95 \% \rightarrow 95 \% \rightarrow 100 \% \rightarrow 100 \%) 45$ $\mathrm{min} / 1 \mathrm{~h} \rightarrow$ second $\rightarrow$ xylene xylene $1 \mathrm{~h} \rightarrow$ paraffin soaked three times, 45 min each time $\rightarrow$ paraffin pack buried $\rightarrow 3 \mu \mathrm{m}$ sections.

HE staining l) dewaxed to water: xylene $10 \mathrm{~min} \times 2 \rightarrow$ graded alcohol $(100 \% \rightarrow 100 \% \rightarrow 95 \% \rightarrow 95 \% \rightarrow 90 \%$ $\rightarrow 80 \% \rightarrow 70 \%) 2 \mathrm{~min} /$ time; 2) rinsed under running water for a few min; 3) immersed in hematoxylin dye dip $5 \mathrm{~min}$; 4) differentiated $5 \mathrm{~s}$ in hydrochloric acid alcohol $\rightarrow$ water washed $5 \mathrm{~min}$; 5) dye eosin stained $3 \mathrm{~min} \rightarrow$ water washing $3 \mathrm{~min}$; 6) dehydrated: graded alcohol $(95 \% \rightarrow 95 \% \rightarrow 100 \% \rightarrow 100 \%) 2 \mathrm{~min} /$ time $\rightarrow$ xylene $30 \mathrm{~s}$; 7) dried, mounted with neutral gum, the results observed. HE staining Results found: blue-black nuclei, cytoplasm pale red.

\subsubsection{SP Immunohistochemical Labeling Experiment Steps}

Specimens of gastric mucosa were fixed in the $4 \%$ paraformaldehyde, paraffin-embedded, sliced; the slides were under anti-tablet and anti-fish treatment after the biopsy, the glass film were put into the incubator $\left(58^{\circ} \mathrm{C}\right.$ $60^{\circ} \mathrm{C}$ ) for 60 min to make the slice close adhesion; dewaxing: slide dewaxed in xylene, 2 times per $8 \mathrm{~min}$; closed endogenous peroxidase: $30 \% 10 \mathrm{~A}+\mathrm{H}_{2} \mathrm{O}_{2}$ mixture of distilled water at room temperature for $10 \mathrm{~min}$ to close the retention of endogenous peroxidase; washed in distilled water three times; high repair antigen: the slides immersed in $0.01 \mathrm{M}$ citrate buffer (PH6.0), high-pressure furnace heating with the interval 50 - 60 min, jetted 1 - 2 
min. Cooling for the next step; dropping 5\% BSA blocking solution at room temperature for $20 \mathrm{~min}$. Rejection to the excess liquid, do not wash; dropping TFF1, TFF2, TFF3 first antibody $50 \mu \mathrm{L}, 4^{\circ} \mathrm{C}$ overnight; PBS wash 3 min $\times 3$ times, drops biotinylated goat anti-mouse IgG 50 $\mu \mathrm{L}$, incubated at room temperature for $10 \mathrm{~min}$; PBS wash 3 min $\times 3$ times, drop a streptomycin avidin horseradish peroxidase, incubated at room temperature $10 \mathrm{~min}$; PBS wash $3 \mathrm{~min} \times 3$ times, each slice plus $100 \mathrm{uL}$ freshly prepared DAB dissolved under the control of reaction time, generally 5 - 30 min; termination of color, distilled water; Mayer hematoxylin contrast staining 20 s, running water; $1 \%$ hydrochloric acid alcohol differentiation $1 \mathrm{~s}$, running water 5min; 95\%, 100\% ethanol dehydration, drying, dropping xylene, neutral resin were mounted.

SP immunohistochemical staining results found: the cytoplasm or cell membrane showed positive brown particles. Image analysis: random selection of 20 glands, Motic Imaged Advanced 3.0 image analysis software to detect the positive signal average optical density values to reflect the strength of TFF expression. The higher optical density, TFF expression will beStatistical.

\subsubsection{Statistical Analysis}

The relative expression level of each sample of target gene was calibrated by reference gene with mean $\pm S D$, with SPSS13.0 for Windows software package for statistical data, by using single factor analysis of variance, Comparison withSNK each other.

\section{RESULTS}

\subsection{Observation of Rat Gastric Tissue}

In the normal group, gastric mucosa was pink, smooth, shiny and covered with much mucus, the surface of the plica which showed regular wrinkles was smooth, the stomach wall was elastic; in the model group, the mucous was thin and gray, the plica was flat and exhibited on the surface irregular wrinkles, which were rare or may be absent in some cases, the stomach wall lacked elasticity; as for Anwei decoction and Fuchun groups, parts of the gastric mucosa returned to normal, which was obvious in Anwei decoction group.

\subsection{Observation with Optical Microscope}

Normal group: Gastric mucosa was thick, with normal structure in all layers; its epithelial cells were regularly arranged; its inherent glands were in regular shape and well organized, showed no phenomenon of being atrophic, deciduous or defective; a few eosinophilic granulocytes were found in it (Figure 1(A)). Model group: Gastric mucosa became significantly thinner than the normal one, came off or eroded on local focuses; its proper glands decreased markedly quantitatively, arranged sparsely and irregularly, reduced in volume, and expanded like a sac individually; lymphocytes infiltrated in the proper layer of the mucous membrane, in parts of which lymph follicles were seen (Figure 1(B)). Anwei decoction group: the mucosa showed normal structure in every layer, its proper glands were large in number and arranged regularly, its proper layer exhibited a bit infiltrated cells (Figure 1(C)). Weifuchun group: slightly thinner than the normal one, the epithelium of gastric mucous membrane was arranged regularly, showed no obvious defects; the proper glands was slightly disordered, parts of them were reduced in volume, but a few of them were expanded; lymphocytes were found infiltrated in the proper layer (Figure $1(\mathrm{D})$ ). These results suggest that both Anwei decoction and Weifuchun could improve the pathomorphological changes of the gastric mucosa, the effect of Anwei decoction being better.

\subsection{The Effect of Anwei Decoction on the Expression of TFF1, TFF2 and TFF3 Proteins in Gastric Mucous Membrane of CAG Rat Models}

\subsubsection{The Effect of Anwei Decoction on TFF1 Protein Expression in Gastric Mucous Membrane of CAG Rat Models}

In Normal group, the expression of TFF1 protein was weakly positive, it mainly occurred in the cytoplasm of the neck of gastric glands; cells involving the positive expression were chiefly parietal cells and cells in the neck of the gastric mucous membrane. In Model, Weifuchun and Anwei decoction groups, TFF1 protein expression in gastric mucosa cells was increasingly positive, some being strong (see Figure 2).

Compared with Model and Normal groups, the optical density of TFF1 protein expression in gastric mucosa

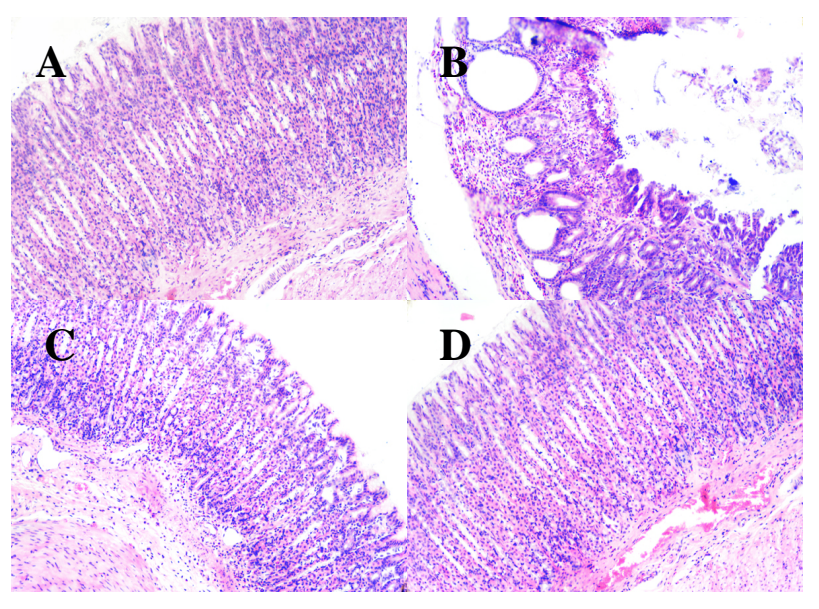

Figure 1. Optical microscope view of the gastric mucosa (HE staining $\times 10$ ). A: normal group; B: model group; C: Anwei decoction group; D: stomach Fu-chun group. 


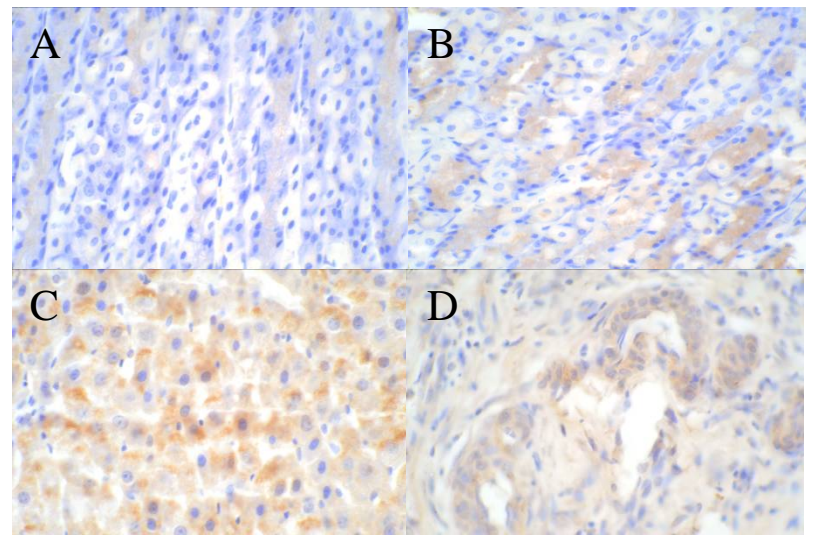

Figure 2. TFF1 expression in gastric mucosa epithelial cells of rats of four groups. A: normal group; B: model group; C: Anwei decoction group; D: stomach Fu-chun group $(\times 40)$.

cells of Anwei decoction and Weifuchun groups was notably increased $(\mathrm{P}<0.01)$, and that of Anwei decoction group was significantly different from that of Weifuchun group $(0.54 \pm 0.07$ VS $0.47 \pm 0.06, P<0.01)$ (Table 1$)$.

\subsubsection{The Effect of Anwei Decoction on TFF2 Protein Expression in Gastric Mucous Membrane of CAG Rat Models}

In Normal group, the expression of TFF2 protein was weakly positive, it mainly occurred in the cytoplasm and cell membranes (primarily in the latter) of the neck and base of gastric glands; cells involving the positive expression were chiefly parietal cells and cells in the neck of the gastric mucous membrane. In Model, Low-dose Anwei decoction, Middle-dose Anwei decoction, Weifuchun and Large-dose Anwei decoction groups, cells participating in TFF2 protein expression gradually increased, some expression being strong (see Figure 3).

Compared with Model and Normal groups, the optical density of TFF1 protein expression in gastric mucosa cells of Anwei decoction and Weifuchun groups was markedly increased $(\mathrm{P}<0.01)$, and that of Anwei decoction group was significantly different from that of Weifuchun group $(0.53 \pm 0.06$ vs $0.46 \pm 0.06, \mathrm{P}<0.01)$ (Table 1).

\subsubsection{The Effect of Anwei Decoction on TFF3 Protein Expression in Gastric Mucous Membrane of CAG Rat Models}

In Normal group, the expression of TFF3 protein was weakly positive, it mainly occurred in the cytoplasm of the neck and base of gastric glands; cells involving the positive expression were chiefly parietal cells and cells in the neck of the gastric mucous membrane. In Model, Low-dose Anwei decoction, Middle-dose Anwei decoction, Weifuchun and Large-dose Anwei decoction groups, cells concerning TFF3 protein expression became increasingly decreased, but the expression in these cells was stronger than in cells of the Normal group (see Figure 4).

The optical density of TFF3 protein expression in gastric mucosa cells of Anwei decoction and Weifuchun groups was obviously lower than that of Normal group (P $<0.01$ ); no significant differences existed between Anwei decoction and Weifuchun groups $(0.37 \pm 0.05$ vs $0.41 \pm 0.07, \mathrm{P}>0.05$ ) (Table 1).

Table 1. Influence of Anwei decoction on the optical density of TFF protein expression in gastric mucosaof CAG rats $(n=10$, mean $\pm \mathrm{SD}$ ).

\begin{tabular}{cccc}
\hline Group & TFF1 & TFF2 & TFF3 \\
\hline normal control group & $0.16 \pm 0.06$ & $0.14 \pm 0.04$ & $0.15 \pm 0.06$ \\
pathologic model group & $0.38 \pm 0.04^{\mathrm{b}}$ & $0.37 \pm 0.05^{\mathrm{b}}$ & $0.53 \pm 0.07^{\mathrm{b}}$ \\
Anwei Decoction group & $0.54 \pm 0.07^{\text {bdf }}$ & $0.53 \pm 0.06^{\text {bdf }}$ & $0.37 \pm 0.05^{\text {bd }}$ \\
Weifuchun group & $0.47 \pm 0.06^{\text {bd }}$ & $0.46 \pm 0.06^{\text {bdc }}$ & $0.41 \pm 0.07^{\text {bdc }}$ \\
\hline
\end{tabular}

Compared with normal group, ${ }^{\mathrm{a}} \mathrm{P}<0.05$, ${ }^{\mathrm{b}} \mathrm{P}<0.01$; compared with model group, ${ }^{\mathrm{C}} \mathrm{P}<0.05$, ${ }^{\mathrm{d}} \mathrm{P}<0.01$; with Weifuchun group, ${ }^{\mathrm{e}} \mathrm{P}<0.05,{ }^{\mathrm{f}} \mathrm{P}<0.01$.

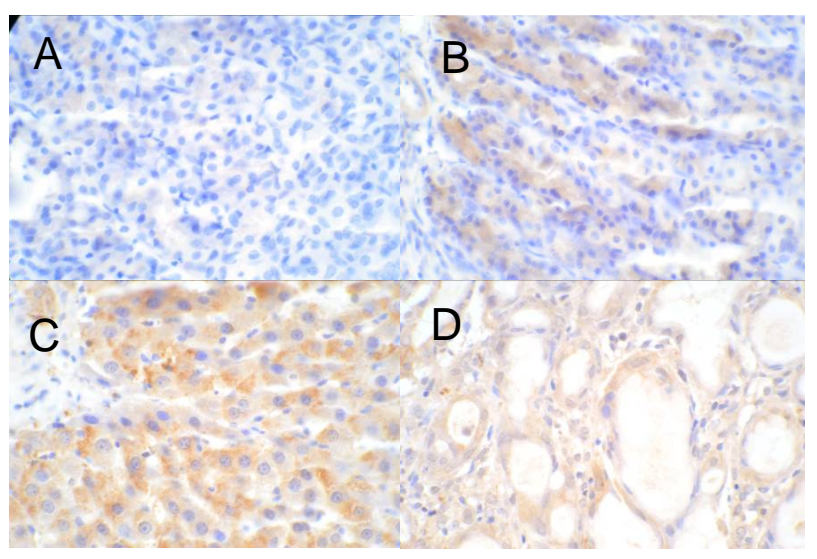

Figure 3. TFF2 expression in gastric mucosa epithelial cells of rats of four groups. A: normal group; B: model group; C: Anwei decoction group; D: stomach Fu-chun group $(\times 40)$.

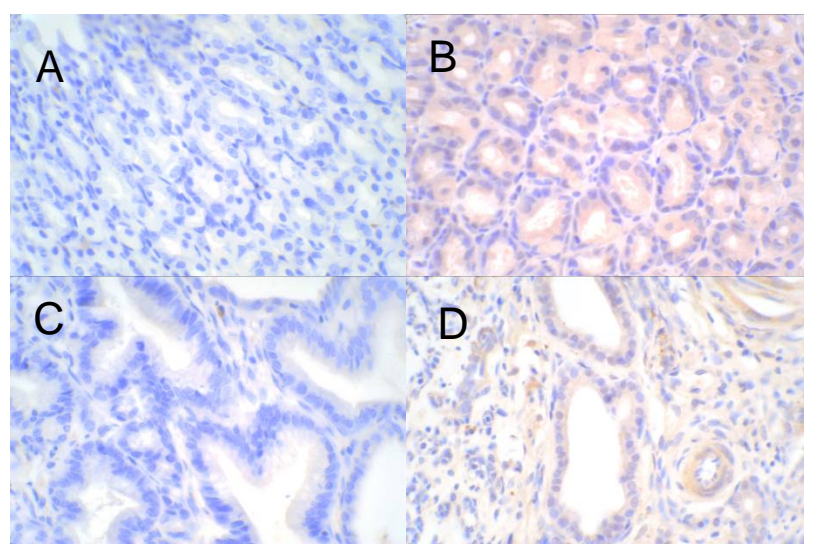

Figure 4. TFF3 expression in gastric mucosa epithelial cells of rats of four groups. A: normal group; B: model group; C: Anwei decoction group; D: stomach Fu-chun group $(\times 40)$. 


\section{DISCUSSION}

CAG is very similar to "distention and fullness", "epigastric pain”, "gastric discomfort” and other diseases of traditional Chinese medicine (TCM) in symptoms. Its pathogenesis mainly includes weakness of the spleen and stomach and stomach Qi rising, which are accompanied by weakness of Qi and Yin and damp stagnation or damp-heat and blood stasis. Clinically, manifestations of CAG generally comprise deficiency mixed with excess and cold mixed with heat. Organs showing pathological changes in CAG involve the spleen, stomach and liver. Anwei decoction is prepared based on the proved recipe formulated and used to treat chronic gastric disease by Professor Lin Peixiang, a national famous doctor of TCM from Guangxi Traditional Chinese Medical University [3]. With a strict formulation and a unique and significant curative effect, the decoction is made from drugs which are cold and hot in nature and can invigorate the circulation of blood and Qi. Weifuchun tablet can Jianpi Yiqi, Huoxue Jiedu, precancerous lesions of gastric cancer indications, is approved by the drug regulatory department of the treatment of precancerous lesions of gastric cancer, the protection of varieties of traditional Chinese medicines, the drug effect, is mainly composed of water chestnut, three seven, Fructus aurantii and strengthening the spleen and replenishing Qi drugs, can improve blood circulation, eliminate inflammation lesions, promote gastric mucosal regeneration [5].

The results show that after being treated with Anwei decoction and Weifuchun, the experimental CAG rats were improved in the following ways: the gastric mucosa became thicker, the number of proper glands was significantly increased, the glands got larger in size. After treatment, rats in Anwei decoction group had gastric mucosa which was normal in every layer of its structure except a few infiltrated cells were seen in the proper layer, and whose proper glands were rich (not decreased significantly compared with those in normal rats) and regularly arranged; rats in Weifuchun group showed slightly thin gastric mucosa whose epithelial cells were organized regularly and had no obvious defects, and whose proper glands, some of which were reduced in volume and a few of which were expanded in size, were somewhat irregularly arranged, this gastric mucosa also exhibited infiltrated cells in the proper layer. These results indicate that both Anwei decoction and Weifuchun can improve the pathomorphism of gastric mucosa, Anwei decoction having better effects than Weifuchun.

TFF is a group of small molecule polypeptides with a specific trefoil domain synthesized and secreted by the gastrointestinal mucus-secretion epithelia, in mammals consisting of three factors: breast cancer-related peptide (TFF1/PS2), spasmolytic polypeptide (TFF2/SP) and in- testinal trefoil factor (TFF3/ITF). TFF has a strong ability to promote proliferation and migration of epithelial cells, can promote the reconstruction of the damaged area and accelerate epithelial cell migration speed [2]. Researchers have found that the expression of TFF1 becomes gradually decreased during the pathological course from intestinalisation to atypical hyperplasia and at last to gastric cancer in human stomachs [6,7]. In vitro experiments showed that [8], the lowering and recovery of TFF2 protein expression in gastric cancer cells do not affect the nature of gastric cancer cells, and the loss of TFF2 protein expression may be an early event of the formation of gastric cancer; TFF2 also has a protective action on normal gastirc mucosa. TFF3 involves in the regeneration and repair of damaged mucosa and possesses a protective effect on gastrointestinal mucosa, its expression becomes more obvious when gastric mucosal damage is more severe. The underlying mechanism is that TFF3 can accelerate the healing of gastrointestinal epithelium; by interaction or cross-linking, TFF3 can form a viscoelastic mucus gel layer with the mucus glycoprotein to prevent stomach mucosal damage caused by such factors as pepsin and mechanical stress changes and enhance the protection ability of gastrointestinal mucosa as a barrier [9]. Once the repair is completed, receptors on the side of the base cease to contact with trefoil peptides, and cells stop moving. TFF3 plays a significant role in the early regeneration and repair of the gastrointestinal mucosa. Its expression gradually increases from intestinal metaplasia to dysplasia and to gastric cancer [6]. Studies [10,11] also showed that TFF3 expression gets increased from superficial gastritis to atrophic gastritis and to dysplasia, and that TFF3 expression of gastric cancer is lower than that of atrophic gastritis and dysplasia (no significant differences exist between every two of these three groups, but their TFF3 expression is significantly higher than that of the normal group and superficial gastritis).

Our results show that compared with rats in the Model and Normal groups, rats in Anwei decoction and Weifuchun groups had a higher optical density of gastric mucosa TFF1 and TFF2 expression $(\mathrm{P}<0.01)$ and that optical density of Anwei decoction group was significantly different from that of Weifuchun group $(\mathrm{P}<0.01)$. Meanwhile, compared with the Model group, Anwei decoction and Weifuchun groups showed a more notable decrease in optical density of gastric mucosa TFF3 ( $\mathrm{P}<$ 0.01 ), and there was no significant discrepancy between Anwei decoction group and Weifuchun group $(\mathrm{P}>0.05)$. Some scholars [12] in Japan discovered Serum levels of TFF3 are a better marker of gastric cancer than pepsinogen; a testfor the combined levels of serum pepsinogen and TFF3 could improve gastric cancer screening. Somebodys [13] reported that reduced expression of TFF1 and 
increased expression of TFF3 may play a role in the carcinogenesis of gastric cancer. It consistented with our report.

\section{CONCLUSION}

In short, we believe that Anwei decoction can promote cell proliferation and migration, facilitate epithelial cell reformation and quicken epithelial cell migration in the damaged area by enhancing protein expression of gastric mucosa TFF1; on the other hand, through increasing protein expression of gastric mucosa TFF2, the decoction can help form a viscoelastic mucus gel layer, thereby enhancing the protection ability of the gastrointestinal mucosa as a defense barrier and reaching the goal of prevention and control of CAG. According to the mechanism of gastric mucosa protection by TFF3, we hypothesized that TFF3 protein is over expressed in the early process of treating CAG with Anwei decoction; as the condition improves, TFF3 protein expression decreases. During this process, TFF3 protein participates in the regeneration and repair of mucosal injury. Despite our findings in this study, we still know little about the interaction among TFF1, TFF2 and TFF3 proteins in the course of treating CAG with Anwei decoction, so this needs to be further explored.

\section{ACKNOWLEDGEMENTS}

The study was supported by the National Key Technology R\&D Program during the $11^{\text {th }}$ Five-year Plan Period, No.2007BAIO7A17-02; and the Natural Science Foundation of Guangxi, Gui Ke Zi No. 0728187.

\section{REFERENCES}

[1] Gastroenterology Branch of Chinese Medical Association (2006) Consensus on chronic gastritis in China (Part I) (2006, Shanghai.) Chinese Journal of Digestion, 27, 4549.

[2] de Paulsen, N., Brychzy, A., Fournier, M.-C., et al. (2001) Role of transforming growth factor-alpha in von Hippel-Lindau (VHL)-/-clear cell renal carcinoma cell proliferation: A possible mechanism coupling VHL tumor suppressor inactivation and tumorigenesis. Proceedings of the National Academy of Sciences of the United States of America, 98, 1387-1392.

[3] Lin, S. (2001) Clinical series of 100 traditional Chinese medical doctors in 100 years-Lin Peixiang. China Traditional Chinese Medicine Press, Beijing, 10, 99-117.

[4] Wang, S.W., Shi, X.Y. and Huang, C.G. (2001) Pharmacodynamics research and evaluation of traditional Chinese medicine. Shaanxi Science and Technology Press, Xi’an, 315-317.

[5] Xu, W.W. and Wang, M.Q. (2003) Observation of the curative effects of Weifuchun Tablets on chronic atrophic gastritis. Zhejiang Journal of Combination of Chinese Traditional and Western Medicine, 13, 505-506.

[6] Poulsen, S.S., Kissow, H., Hare, K., Hartmann, B. and Thim, L. (2005) Luminal and parenteral TFF2 and TFF3 dimer andmonomer in two models of experimental colitis inthe rat. Regulatory Peptides, 126, 163-171. http://dx.doi.org/10.1016/j.regpep.2004.09.007

[7] Leung, W.K., Yu, J., Chan, F.K., To, K.F., Chan, M.W., Ebert, M.P., Ng, E.K., Chung, S.C., Malfertheiner, P. and Sung, J.J. (2002) Ex-pression of trefoil peptides (TFF1, TFF2, and TFF3) in gastric carcinomas, intestinal metaplasia , and non-neoplastic gastric tissues. The Journal of Pathology, 197, 582-588. http://dx.doi.org/10.1002/path.1147

[8] Suárez, C., Vizoso, F., Rodríguez, J.C., García, I., Raigoso, P., Allende, M.T., García-Muíz, J.L. and GarcíaMorán, M. (2001) Prognostic significance of cytosolic pS2 protein con-tent in gastric cancer. The International Journal of Biological Markers, 16, 37-44

[9] Shiemann, U., Konturek, J., Assert, R., et al. (2002) mRNA expression of EGF receptor ligands in atrophic gastritis before and after helicobacter pylori eradication. Medical Science Monitor, 8, 53-58.

[10] Alison, M.R., Chinery, R., Poulsom, R., et al. (1995) Experiment alulceration leads to sequential expression of spasmolytic polypeptide, intestinal trefoil factor, epidermal growth factor and transforming growth factor alpha mRNAs in rat stomach. The Journal of Pathology, 175, 405-414. http://dx.doi.org/10.1002/path.1711750408

[11] Nie, S.N., Sun, H.C., Wu, X.H., et al. (2005) The regulation of trefoil peptides on gastric adaptive cytoprotection. Chinese Critical Care Medicine, 17, 302

[12] Susumu, A.K., Yasukazu, O., Toshiaki, G., et al. (2011) Tests for serum levels of trefoil factor family proteins can improve gastric cancer screening. Gastroenterology, $\mathbf{1 4 1}$ 837-845, e7.

[13] Im, S., Yoo, C.Y., Jung, J.-H., et al. (2013) Reduced expression of TFF1 and increased ex-pression of TFF3 in gastric cancer: Correlation with clinicopathological parameters and prognosis. Inter-national Journal of Medical Sciences, 10, 133-140. 